\title{
Distress in neurocognitive disorders due to Alzheimer's disease and stroke
}

\author{
CAMELIA MARIA DINDELEGAN ${ }^{1}$, DARIAN FAUR ${ }^{1}$, \\ LAVINIA PURZA $^{2}$, ADRIAN BUMBU $^{3}$ and MONICA SABAU ${ }^{3,4}$ \\ ${ }^{1}$ Psychology Department, Faculty of Social Humanistic Science, University of Oradea, 410087 Oradea; \\ ${ }^{2}$ Department of Pharmacy, Faculty of Medicine and Pharmacy, University of Oradea, 410028 Oradea; \\ ${ }^{3}$ Department of Psycho-Neurosciences and Rehabilitation, Faculty of Medicine and Pharmacy, \\ University of Oradea, 410068 Oradea; ${ }^{4}$ Clinical Department of Neurology, \\ Emergency Clinical County Hospital, 410169 Oradea, Romania
}

Received March 06, 2020; Accepted April 06, 2020

DOI: $10.3892 /$ etm.2020.8806

\begin{abstract}
Patients with neurocognitive disorders experience subjectively the concept of quality of life; this is the reason why researchers avoid approaching this concept and prefer to focus attention on the emotional profile of the caregivers. Many studies highlight the efforts both emotional and financial made by caregivers in case of patients diagnosed with neurocognitive disorders. The present study shows the differences between the patients diagnosed with neurocognitive disorder due to Alzheimer's disease and patients diagnosed with stroke, as well as the Romanian norms for the short form of Geriatric Depression Scale. The study group consisted of the clinical population ( $\mathrm{N}=45)$, selected according to the inclusion/exclusion criteria, following the principles of Helsinki Declaration for Ethical Medical Research. The study was conducted at the Neuropsychiatry section of the Municipal Clinical Hospital, Dr Gavril Curteanu, Oradea, Romania. The results showed significant differences between the two types of patients in terms of quality of life, $\mathrm{t}(43)=-7.99, \mathrm{P}=0.001$, affective distress, $\mathrm{t}(43)=5.10, \mathrm{P}=0.001$ and perceived stress, $\mathrm{t}(43)=3.81, \mathrm{P}=0.001$. The internal consistency of the scale is high, the coefficient KR-20 being 0.86 .
\end{abstract}

\section{Introduction}

Quality of life is a concept often avoided by researchers because of its subjective experimentation by patients with neurocognitive disorders, researchers prefer to focus on the emotional profile of the caregivers. The studies show that, for

Correspondence to: Ms. Lavinia Purza, Department of Pharmacy, Faculty of Medicine and Pharmacy, University of Oradea, 1 December Sq. 10, 410028 Oradea, Romania

E-mail:purza_lavinia@yahoo.com

Key words: Alzheimer's disease, stroke, neurocognitive disorders, distress, Geriatric Depression Scale, cognitive impairment a person diagnosed with such a disorder, the caregivers have to make sustainable efforts, both financially and emotionally (1-3).

Alzheimer's disease (AD), the most common onset of adult dementia, is a neurodegenerative disease characterized by loss of performance capacity and of dispositional and behavioural intellectual functioning (4). Since 1906 (when Alois Alzheimer first observed this form of disease) until now, researchers have suggested several factors that could be considered to determine the onset of the disease. In a 2009 report, Mucke indicated certain non-genetic factors that could increase the risk of developing dementia due to AD: low education, obesity, diabetes or cranio-cerebral trauma (5). However, the strongest arguments for elements that can cause the disease revolve around genetic inheritance. In this respect, the apolipoprotein E4 (ApoE4) polymorphism increases the risk of disease (4). This protein has the role of transporting cholesterol through the blood and binds to the amyloid protein, playing a role in regulating the latter.

Dementias due to neurodegenerative diseases are common in the elderly population, but at the same time, vascular pathology is also widespread in the same age groups (6). The main cause of disability after the age of 65 is represented by stroke or cerebrovascular accident (CVA) and the patients' sequelae demand long periods of care and recovery. This pathology is considered to become the main cause of mortality until 2030 (7-9). The causes of developing dementia on stroke background are not exactly known. About a quarter of the population diagnosed with a stroke form develops dementia as well (10). Thus, if the stroke occurs in an area important for cognitive functions, such as thalamus, then it will certainly be followed by the decline of the affected cognitive function, fact that coincides with dementia. At the mechanism level, vascular dementia involves a break between the connections of the axons that communicate between the two cerebral hemispheres and those that link the cerebral cortex with the grey matter, but a causal rather than a descriptive explanation could not be formulated (10). Reported data indicate an association between $\mathrm{AD}$ and stroke, the connection between $\mathrm{AD}$ and atherosclerosis, regarding comorbidities, data detected 
in the brains of patients with AD in the 30-60\% who have a wide variety of lesions, the poor clearance of amyloid in $\mathrm{BBB}$, cognitive impairment present up to $100 \%$ in $\mathrm{AD}$ brain and associated CAA, significant cerebral microangiopathy. Regarding the chronic neurodegeneration in $\mathrm{AD}$, the vascular disorders present essential characteristics (11). Thus, an important role in the pathogenesis of AD may be attributed to neurovascular dysfunction (11). There is evidence that the association between AD-type pathology and the circle of Willis atherosclerosis are interconnected and suggests that pathophysiological mechanisms that are mutually synergistic or physiologically common etiologically contribute to both pathologies (11).

The specialized literature that differentially analyses the category of patients with AD and that of the patients with vascular dementia is poorer, both quantitatively and methodologically, especially when talking about the differential analysis of the quality of life, the researchers' option being to treat the subject in parallel. The importance of analysing the subjective dimension of neurocognitive disorders derives from the lack of interventions specifically outlined on the type of disorder because of the lack of information about the disease experimenting at the intrapsychic level. Thus, Cohen showed that the effect of the lack of such studies materializes in faddy psychological, behavioural, but also familial therapeutic interventions, without sustainable empirical support and without improvement focused on the problem, to transform them into efficient working tools. Due to the lack of the subjective experience knowledge, the clinical decisions of the specialists who have to deal directly with the problem of neurocognitive disorders will not represent the patient's needs in a fair manner (12).

The purpose of this study was to carry out an evaluation of the differences between the two types of dementia, evaluation that would be the basis for further research regarding the development of an intervention program that would follow the specifics of each group of subjects.

\section{Patients and methods}

Objectives. The present report was set out to check whether there are differences in the perception of quality of life on the sub-dimension of well-being in patients diagnosed with AD compared with those diagnosed with vascular dementia. At the same time, it is aimed to observe any differences that could occur between the same groups of subjects on the dimension of the Profile of Affective Distress (PAD) and its 7 factors, namely the score of global distress, functional sadness, dysfunctional depression, functional worry, non-functional anxiety, total functional score and total dysfunctional score. Also, it analysed the relationship between the two groups regarding the level of perceived stress, assuming that there are differences in this regard.

A final goal was the adaptation to the Romanian clinical population of the Geriatric Depression Scale - Short Form (GDS-SF) 15. This scale is an effective tool for identifying depression in the elderly, especially in the clinical context, where depression appears as a comorbidity to another condition. The authors recommend applying the scale to elderly patients with dementia or other physical impairment, which could speed up the fatigue process. This scale was adapted for the Romanian population in 2017 (13). The difference is that the population the authors evaluated was the normal elderly population, while this study refers to the clinical population. As mentioned, it is intended to capture the effectiveness of the scale in detecting the symptomatic elements of depression in the elderly who have a certain level of cognitive impairment.

Hypotheses. The hypotheses are described in Table I, given that the design was unifactorial intergroup and the independent variable was the same in all three cases: type of dementia (Alzheimer's, vascular).

Participants. The study was conducted in accordance with the WMA Declaration of Ethical Helsinki - Medical Research Involving Human Principles for Subjects. All the participants provided a signed written informed consent and accepted to take part in the research. Ethics approval was received from the Medical Center Crucea Alba 'Dr Oros si asociatii', Oradea, Romania (no. 25/Sept. 11, 2019). The participants in this study were selected primarily based on the neurocognitive disorder they suffered from: patients with neurocognitive disorder due to $\mathrm{AD}$, respectively, neurocognitive disorder due to stroke. A secondary, but equally important criterion according to which the group was outlined was the patients' score in the Mini Mental State Examination (MMSE) test designed in 1975 by the psychiatrist Folstein to indicate, in the clinical practice, the level of cognitive deterioration of the patient. Thus, for this study only the patients who obtained scores that could be classified in the categories of mild and moderate cognitive impairment were selected, eliminating those with severe deterioration, because the concepts approached in this investigation are no longer relevant for this category, the cognitive impairment being greatly accentuated. In most cases the MMSE scores were taken from the consultation register of the clinical psychologists of the hospital who examined these patients on admission, but in a few cases, they were obtained by administering the MMSE to the respective patients.

After applying the sampling criteria, patients who were within the limits prescribed by the criteria were randomly selected. The participants in this study were patients from the White Cross Medical Centre, or former patients who had at least one consultation in the Medical Centre, with specific psychiatry, neurology and psychology. There were 22 patients suffering from neurocognitive disorder due to $\mathrm{AD}$ and 23 patients with secondary neurocognitive disorder due to stroke. Of the total subjects that were included in the group suffering from neurocognitive disorder due to AD, $16(72.73 \%)$ were females, while $6(27.27 \%)$ were males. The age of the participants ranged from 53 to 81 years. Regarding the subjects who participated in the group of patients suffering from stroke secondary neurocognitive disorder due to stroke, $14(60.87 \%)$ were females and $9(39.13 \%)$ males. Their ages ranged from 54 to 84 years.

Therefore, a total of 45 subjects between the ages of 53 and 84 participated in this study, where $30(66.67 \%)$ were females and $15(33.33 \%)$ males. Data on their marital status, occupation (for those who did not have retirement age) or other known general medical conditions were not collected for the present study, but most patients listed certain elements 
Table I. Study hypotheses.

Items

Hypothesis 1

Supposition

Dependent variable

Differences regarding the perception of quality of life in patients with AD versus patients with stroke Perception of the quality of life operationalized by the score obtained by applying the WHO Questionnaire of Well-being.

Items

Hypothesis 2

Supposition

Dependent variable

Differences regarding the profile of affective distress in AD patients versus patients with stroke

Affective distress profile measured using the PAD Scale.

Items

Hypothesis 3

Supposition

Dependent variable

Differences regarding perceived stress in AD patients versus patients with stroke

Level of perceived stress measured by applying the PSQ.

AD, Alzheimer's disease; PAD, Profile of Affective Distress; PSQ, Perceived Stress Questionnaire.

of their personal history before applying the tests. It is easy to understand that in the elderly participants who also suffer ed from other medical conditions or who no longer benefited from socio-family support, either due to the death of their life partner or because of alienation from children, these parasitic variables interfered to some extent with the areas examined in this study, even though they were formulated in such a way as to focus on the effects of the main disease they suffered from.

\section{Instruments}

Geriatric Depression Scale, short form. In addition to the differences between the two groups of subjects included in the study, it is intended to adapt for the Romanian clinical population the GDS, conceived in its long form in 1982 by Yesavage and collaborators, being a scale of 30 items that allowed the differentiation of depressives from non-depressives among the elderly population. The subjects answered 'Yes' or 'No' to each of the 30 questions, then the total score was cumulated and interpreted. A score between 0 and 12 was considered normal by authors, while scores above 12 fell into the depression category.

Regarding the short form of the scale, items 1, 5, 7, 11 and 13 , if receiving a positive answer, indicate the absence of depression. The total score is calculated by summing all the answers 'No' for the aforementioned items and the answers 'Yes' for all the other items. A score between 0 and 5 indicates the absence of depression, for a score ranging from 5 to 10 , detailed examination is proposed, being an interval suggestive for depression, and scores $>10$ indicate the presence of depression in overwhelming proportion. This scale was adapted for the Romanian population in 2017 (13).

WHO 5 Well-Being Index. The Well-Being Index evaluates in only 5 items the subjective well-being state reported by the subject. Applying it takes $2-3 \mathrm{~min}$, each of the 5 items being evaluated on a Likert scale in 6 steps (0), absent all the time; 5 , present all the time). For the rating, the scores of each item are summed resulting an overall score ranging from 0 to 25 .
Scores having values $<12$ suggest a very low well-being and testing for depression is recommended. The psychometric characteristics of the scale corresponding to the Romanian population are given by the adaptation made by Preoteasa \& Preoteasa (14).

Profile of affective distress. This scale measures the functional and dysfunctional negative emotions included in the categories of worry/anxiety, respectively sadness/depression, as well as the positive emotions. Designed in 2005, this scale is based on the items of the Profile of Emotional Distress scale, short version (Profile of Mood Disorders). To the already existing items of the scale were added other items using a dictionary of synonyms, thus obtaining 39 adjectives that describe emotions. The grouping of items on subscales is done as follows: 6 items represent negative functional emotions in the sadness category; 8 items refer to non-functional negative emotions in the depression category; other 6 items are in the category of functional negative emotions of the concern type; 6 items are in the category of non-functional negative emotions such as anxiety; 13 items represent positive emotions. At the same time, the scale allows the calculation of a global score of affective distress, as well as a dysfunctional global score, and of a functional global score. Applying it can be done individually or in a group, having as materials paper and pencils. The evaluation is made by assigning numbers from 1 to 5 to each label connected to an emotion - not at all (1), very little (2), medium (3), much (4), very much (5). For a global distress score, the scores of the 26 negative items will be cumulated with the scores of the 13 positive items inversely rated. For a total distress score, only scores from the negative items listed directly are summed. The psychometric qualities of the scale show first and foremost a good internal consistency for each subscale. The coefficient Alpha (Cronbach) varies between 0.80 in the worry (functional) and anxiety (dysfunctional) subscales and 0.94 in the total distress score subscale. The validity of the concept is given by the correlation with 
other instruments that measure similar aspects or even the same dimensions, such as Beck Depression Inventory (BDI) or Dysfunctional Attitude Scale (DAS-A). The scale has a standard in 5 percentile classes (15).

Perceived Stress Questionnaire (PSQ). The questionnaire was developed by Levenstein et al (16) in 1993 and contains 30 items that describe the cognates and emotions that appear as responses to environmental demands that exceed the responsiveness of the subject. The subject gives each item a label ranging from 1 to 4 , namely: almost never (1), sometimes (2), often (3), almost always (4). Items 1, 7, 10, 13, 17, 21, 25 and 29 are marked inversely. The score ranges from 30 to 120 , presented in three groups: low perceived stress (with values between 30 and 60), medium stress (circumscribed between 61 and 90) and high stress (which is in the range 91-120) (16). The initial internal consistency of the scale was very good, with the coefficient Alpha (Cronbach) values $>0.90$. At the same time, the fidelity of the scale was given by the test-retest stability, at 3 months and 1 year. The scale was adapted to the Romanian population (17).

Procedure. Subjects were individually administered each of the 4 questionnaires. They were announced that their answers are given under the protection of anonymity, they only revealing their age at our request. Because each subject was part of a clinical group, the activity was carried out at the Municipal Clinical Hospital, Dr Gavril Curteanu, Department of Neuropsychiatry. The questionnaires were administered using the interview method, as most patients had difficulty getting out of bed, especially those in the group with vascular dementia. In order to avoid fatigue, the clinical observations for questionnaires were also used, especially in the profile of affective distress, where many of the adjectives used were synonyms.

To apply the questionnaires, in most cases the interaction with the subjects was in the wards where they were admitted, excepting the Psychiatry and the Ergo-therapy sections, where some patients came to a separate place to be interviewed, guided by a nurse. Regarding the extraction of the psychometric qualities of the GDS-SF scale after its application on the Romanian clinical population, the application of the scale was carried out concurrently with the other three, it being the last one. To deduce the validity of the concept, the GDS-SF scale was correlated with the well-being scale used in this research. At the same time, the GDS-SF with the PAD were entirely correlated, thus with a global distress score, and then with the subscale of the dysfunctional emotions on the depressive dimension, that is the exact concept validated by the study. The criterion validity of the scale was deduced in the initial adaptation on the Romanian population that did not suffer from cognitive impairment by dividing the patients into 2 groups - one whose subjects met the conditions necessary for diagnosis with major depressive episode according to DSM IV-TR criteria and the other one that was the control group (13). The GDS-SF averages were then compared using the simple t-test. In this case, the target population being those having at least one form of cognitive impairment, the total number of 45 subjects were divided into two groups based on the scores obtained at the subscale of
Table II. Skewness and Kurtosis indicators for well-being, affective distress profile and perceived stress.

\begin{tabular}{lrrr}
\hline $\begin{array}{l}\text { Type of } \\
\text { neurocognitive } \\
\text { disorder }\end{array}$ & Well-being & $\begin{array}{c}\text { Profile of } \\
\text { affective } \\
\text { distress }\end{array}$ & $\begin{array}{c}\text { Perceived } \\
\text { stress }\end{array}$ \\
\hline AD & 22 & 22 & 22 \\
Valid & 0 & 0 & 0 \\
Lack & 0.845 & -0.483 & -0.174 \\
Skewness & 0.410 & -0.887 & -1.073 \\
Kurtosis & & & \\
CVA & 23 & 23 & 23 \\
Valid & 0 & 0 & 0 \\
Lack & -0.485 & 1.009 & 1.693 \\
Skewness & 0.445 & 0.396 & 1.807 \\
Kurtosis & & &
\end{tabular}

AD, Alzheimer's disease; CVA, cerebrovascular accident.

the dysfunctional depression-type emotions within the PAD scale. According to the standard for this subscale, there are no differences between women and men, and an average level of dysfunctional emotions of depression type is equivalent to a score of 18 , the limit score from which it is considered that the dysfunctional emotions of depression type start to have a high level being 26 . Therefore, in order to be able to accurately estimate the difference between depressive and non-depressive patients, for the control group scores were selected that did not reach the threshold of 18 , all the other subjects being included in the depression group.

\section{Results}

The descriptive analysis of the results showed that the data distribution is symmetrical for each group. Thus, for each of the 3 variables proposed for the study, the vaulting and asymmetry indicators had values between -2 and +2 , both for the group with neurocognitive disorder due to AD and for the group of subjects who had neurocognitive disorder due to stroke. Table II shows the exact values of the data distribution indicators, together with the standard errors of each.

In order to test the hypotheses and, implicitly, to compare the two groups proposed for the study based on the mentioned variables, the simple statistical t-test was used, decision made after checking the distribution of data and observing its symmetry. In this respect, for the differences between patients with $\mathrm{AD}$ and those with stroke, regarding the level of well-being, for the Levene test obtained a $\mathrm{F}=0.25, \mathrm{P}>0.05$, statistically insignificant. As a result, the homogeneity of variances was assumed, having no differences between them. The simple t-test obtained a $\mathrm{t}(43)=-7.99, \mathrm{P}<0.001$, statistically significant. There are differences between the patients with neurocognitive disorder due to AD and those with neurocognitive disorder due to stroke, in terms of well-being. The results of the applied statistical test and the direction taken by the differences between the two groups for which the level of 
Table III. Levene test and simple t-test for well-being.

\begin{tabular}{|c|c|c|c|c|c|}
\hline \multicolumn{2}{|c|}{ Levene test } & \multirow[b]{2}{*}{$\mathrm{t}$} & \multirow[b]{2}{*}{ DF } & \multirow[b]{2}{*}{ P-value } & \multirow[b]{2}{*}{ Mean difference } \\
\hline $\mathrm{F}$ & P-value & & & & \\
\hline 0.25 & 0.61 & -7.99 & 43 & $<0.001$ & -9.01 \\
\hline \multicolumn{5}{|c|}{ Means and standard deviation } & \\
\hline Variable & $\begin{array}{l}\text { Type of } \\
\text { disorder }\end{array}$ & $\mathrm{N}$ & Mean & SD & \\
\hline \multirow[t]{2}{*}{ Well-being } & $\mathrm{AD}$ & 22 & 7.5909 & 4.05509 & \\
\hline & CVA & 23 & 16.6087 & 3.49986 & \\
\hline
\end{tabular}

DF, degrees of freedom; AD, Alzheimer's disease; CVA, cerebrovascular accident.

Table IV. Levene test and simple t-test for affective distress.

\begin{tabular}{|c|c|c|c|c|c|}
\hline \multicolumn{2}{|l|}{ Levene test } & \multirow[b]{2}{*}{$\mathrm{t}$} & \multirow[b]{2}{*}{$\mathrm{DF}$} & \multirow[b]{2}{*}{ P-value } & \multirow[b]{2}{*}{ Mean difference } \\
\hline $\mathrm{F}$ & P-value & & & & \\
\hline 0.552 & 0.462 & 5.108 & 43 & $<0.001$ & 34.94071 \\
\hline \multicolumn{5}{|c|}{ Means and standard deviation } & \\
\hline Variable & $\begin{array}{l}\text { Type of } \\
\text { disorder }\end{array}$ & $\mathrm{N}$ & Mean & SD & \\
\hline Profile of affective distress & $\mathrm{AD}$ & 22 & 119.6364 & 19.82434 & \\
\hline CVA & 23 & 84.6957 & 25.56175 & & \\
\hline
\end{tabular}

DF, degrees of freedom; AD, Alzheimer's disease; CVA, cerebrovascular accident.

well-being was compared can be traced in Table III. According to Table III, patients suffering from AD $(\mathrm{M}=7.59)$ have a much lower perceived well-being compared to those suffering from a neurocognitive disorder due to stroke $(M=16.60)$. Cohen's coefficient $d$ has a value of 2.38 , which shows a very important effect size (the indicators $r$ and $r^{2}$ respectively have values of 0.77 , respectively one $r^{2}=0.59$ ). There were no sex differences regarding the perception of well-being, both women and men presenting equal levels of scores on the well-being scale, both in the AD patients group and in the stroke group.

Regarding the possible differences between the AD and CVA groups on the size of the affective distress profile, following the application of the Levene test, an $\mathrm{F}=0.52$ was obtained, having a significance threshold higher than the minimum limit imposed of 0.05 , the differences between the homogeneities of the variances being insignificant. Therefore, their homogeneity was assumed. After applying the simple t-test, a $\mathrm{t}(43)=5.10, \mathrm{P}<0.001$, statistically significant was obtained (Table IV), suggesting that there are differences between the compared groups in terms of affective distress profile. Thus, patients with AD $(M=119.63)$ tend to have a higher level of global distress compared to patients with stroke (M=84.69). The results of the applied statistical test and the course taken by the differences between the two groups compared for the PAD is illustrated in Table IV.

Related to the effect, Cohen's coefficient $d$ had the value of $1.52\left(r=0.32, r^{2}=0.10\right)$, indicating a very high effect. As the scale for the PAD allows not only to calculate a global distress score, but also to analyse the negative functional and the negative dysfunctional emotions separately, the patients with AD were observed to have a considerably higher level of dysfunctional negative emotion related to anxiety and depression compared to patients who had stroke. The data are summarized in Table V.

Regarding the sex difference, there are no significant differences between men and women in terms of the profile of affective distress, neither in the patients with AD nor in the patients with neurocognitive disorder due to AD. However, a pronounced tendency for difference was manifested between men and women with stroke on the dysfunctional dimension of the anxiety felt $(\mathrm{t}=1.82, \mathrm{P}=0.082)$. Thus, men suffering from a neurocognitive disorder due to stroke tend to be more anxious $(M=11.44)$ than women with the same diagnosis $(M=8.28)$. The differences between the two groups on the 
Table V. Means, Levene test and t-test for dysfunctional emotions.

\begin{tabular}{lccccc}
$\begin{array}{l}\text { Dysfunctional } \\
\text { emotion }\end{array}$ & Pathology & Mean & Levene & $\mathrm{t}$ & P-value \\
\hline Depression & AD & 18.54 & 0.225 & 3.16 & 0.003 \\
& CVA & 12.69 & & & \\
Anxiety & AD & 14.86 & 0.154 & 4.71 & 0.001 \\
& CVA & 9.52 & & &
\end{tabular}

AD, Alzheimer's disease; CVA, cerebrovascular accident.

Table VI. Levene test and t-test for perceived stress.

\begin{tabular}{|c|c|c|c|c|}
\hline \multicolumn{2}{|c|}{ Levene test } & \multicolumn{3}{|c|}{ t-test } \\
\hline $\mathrm{F}$ & $\mathrm{P}$-value & $\mathrm{t}$ & $\mathrm{DF}$ & P-value \\
\hline 0.032 & 0.860 & 3.816 & 43 & $<0.001$ \\
\hline
\end{tabular}

DF, degrees of freedom.

dimension of perceived stress were verified applying the simple t-test. The Levene test showed that there are no differences regarding the homogeneity of variances. Inferential statistics further showed a $\mathrm{t}(43)=3.81, \mathrm{P}<0.001$, statistically significant. Therefore, there are differences in the level of perceived stress in $\mathrm{AD}$ patients compared to those who have suffered a stroke (Table VI). Comparing the means shows that patients suffering from neurocognitive disorder due to AD $(M=65.90)$ will have a higher level of perceived stress compared to patients suffering from neurocognitive disorder due to stroke $(M=50.69)$. The effect size shows a Cohen coefficient $\mathrm{d}$ value of 1.14 that implies a high effect, with important practical applications.

An important element is the sex difference. Basically, there were no differences in perceived stress in men with $\mathrm{AD}$ and those with stroke, $t(13)=1.85, P=0.086$, statistically insignificant. The difference between the two groups included in this research on the perceived stress factor appeared due to the significant differences that appeared between women. Thus, the female patients diagnosed with $\mathrm{AD}(\mathrm{M}=65.18)$ tend to experience a higher level of stress compared to those who have a neurocognitive disorder due to stroke $(M=48.78)$. The result of the $\mathrm{t}$-test for the latter category was $\mathrm{t}(28)=3.43, \mathrm{P}=0.002$, statistically significant.

The internal consistency of GDS-SF indicated by KR-20 is high. As Table VII shows, each of the items on the scale had a high level of fidelity. Elimination of any of them would add, in the best case, 0.08 to the value of the KR-20 coefficient calculated by the inter-correlation of all the 15 items of the scale. Following the correlation of the GDS-SF scale with the well-being scale of Pearson correlation test (since the distributions are symmetrical for the GDS-SF at the population level, the Skewness indicator having the values of -0.636 in the group with $\mathrm{AD}$ and 1.47 in the group with CVA, and Kurtosis
Table VII. Overall mean, scale variance and KR-20 after eliminating some items.

\begin{tabular}{lccc}
\hline Items & $\begin{array}{c}\text { Mean of scale } \\
\text { after removing } \\
\text { the item }\end{array}$ & $\begin{array}{c}\text { Scale variance } \\
\text { after removing } \\
\text { the item }\end{array}$ & $\begin{array}{c}\text { Coefficient KR-20 } \\
\text { after removing } \\
\text { the item }\end{array}$ \\
\hline i1 & 6.2889 & 13.528 & 0.847 \\
i2 & 5.9556 & 15.180 & 0.869 \\
i3 & 6.4667 & 14.664 & 0.860 \\
i4 & 6.0667 & 15.018 & 0.870 \\
i5 & 6.1556 & 13.316 & 0.844 \\
i6 & 6.3778 & 14.149 & 0.855 \\
i7 & 6.1111 & 13.465 & 0.846 \\
i8 & 6.1111 & 13.965 & 0.855 \\
i9 & 6.1111 & 15.510 & 0.877 \\
i10 & 6.2000 & 15.209 & 0.873 \\
i11 & 6.5778 & 16.068 & 0.874 \\
i12 & 6.5778 & 15.204 & 0.862 \\
i13 & 5.9556 & 15.271 & 0.870 \\
i14 & 6.5333 & 14.800 & 0.859 \\
i15 & 6.1556 & 13.362 & 0.845 \\
\hline
\end{tabular}

indicator of -0.267 for those with $\mathrm{AD}$ and 1.36 for those with stroke) a coefficient $r=-0.79, \mathrm{P}<0.001$, statistically significant obtained. Thus, there is a negative correlation between depression and well-being - the higher scale the depression, the lower the well-being and vice versa - as demonstrated by the GDS-SF scale.

Regarding the correlation between GDS-SF and PAD global score, a statistically significant $r=0.83, \mathrm{P}<0.001$ was obtained. At the same time, the correlation between GDS-SF and the depression factor within the PAD shows a coefficient $\mathrm{r}=0.80, \mathrm{P}<001$, statistically significant. The validity of the concept for the scale is deducted from these correlations.

Following the selection made on the basis of the score obtained at PAD depression subscale, in the group of depressive subjects included 17 patients, the rest of 28 being included in the group of non-depressive patients. After checking the symmetry of the data distribution at the population level, the two groups were compared using the simple t-test. Following the analysis of the inferential statistics, the homogeneity of the variances was summed as the $\mathrm{F}$ coefficient of the Levene test equal to $1.99, \mathrm{P}=0.16$, statistically insignificant. The comparison of the means of the two groups showed a $t(43)=6.60$, $\mathrm{P}<0.001$, statistically significant. There are differences between the group of depressive patients, and the control group, of the non-depressive patients, in the direction anticipated - the group with a high score at the depression subscale in the PAD had a much higher average at GDS-SF $(M=10.29)$ compared to the group that had a low score on the same subscale, having a significantly lower average at GDS-SF $(\mathrm{M}=4.42)$. Therefore, the validity criterion of the GDS-SF scale is deduced. The data can be seen in Table VIII.

The magnitude of the effect for this significance threshold is given by the value of Cohen's coefficient $d$. Thus, the coefficient has the value of 2.11 , which suggests a very good effect 
Table VIII. Levene test, t-test and group means for criterion validity.

\begin{tabular}{ccccccc}
\hline $\mathrm{F}$ & P-value & $\mathrm{t}$ & $\mathrm{DF}$ & P-value & Mean depressive patients & Mean non-depressive patients \\
\hline 1.993 & 0.165 & 6.608 & 43 & $<0.001$ & 10.29 & 4.42 \\
\hline
\end{tabular}

DF, degrees of freedom.

Table IX. Descriptive statistics for the sensitivity and specificity of the GDS-SF scale.

\begin{tabular}{|c|c|c|c|}
\hline \multirow[b]{2}{*}{ Patients } & \multicolumn{2}{|c|}{ Reality } & \multirow[b]{2}{*}{ Total } \\
\hline & Real & False & \\
\hline \multicolumn{4}{|l|}{$\begin{array}{l}\text { Classification according } \\
\text { to GDS-SF }\end{array}$} \\
\hline \multicolumn{4}{|l|}{ Depressives } \\
\hline $\mathrm{N}$ & 17 & 0 & 17 \\
\hline $\begin{array}{l}\% \text { of the classification } \\
\text { according to GDS-SF }\end{array}$ & 100.0 & 0.0 & 100.0 \\
\hline$\%$ of reality & 45.9 & 0.0 & 37.8 \\
\hline \multicolumn{4}{|l|}{ Non-depressives } \\
\hline $\mathrm{N}$ & 20 & 8 & 28 \\
\hline $\begin{array}{l}\% \text { of the classification } \\
\text { according to GDS-SF }\end{array}$ & 71.4 & 28.6 & 100.0 \\
\hline$\%$ of reality & 54.1 & 100.0 & 62.2 \\
\hline \multicolumn{4}{|l|}{ Total } \\
\hline $\mathrm{N}$ & 37 & 8 & 45 \\
\hline $\begin{array}{l}\% \text { of the classification } \\
\text { according to GDS-SF }\end{array}$ & 82.2 & 17.8 & 100.0 \\
\hline$\%$ of reality & 100.0 & 100.0 & 100.0 \\
\hline
\end{tabular}

GDS-SF, Geriatric Depression Scale - Short Form.

size, with practical implications of statistical significance regarding the observed differences. The sensitivity and specificity of the GDS-SF scale were also analysed. Before explaining the procedure, it is reminded that the authors of the GDS-SF scale proposed that at a score between 5 and 10, the patient should be carefully examined for depression, as the score has a high probability of being susceptible to depression, and a score $>10$ was considered symptomatic. Thus, to observe the sensitivity and specificity of the scale, the two groups were divided, based on the PAD depression subscale, into depressive and non-depressive patients, to which was added a dichotomous nominal variable where the true or false predictions of the scale were marked according to the standard proposed by authors. Thus, the patients who according to the PAD subscale were in the depressive category and had a score $>5$ in the GDS-SF received the label 'real', while the subjects who were classified in the group of non-depressive, but had a score $>5$ at GDS-SF received the label 'false'. The results of the descriptive analysis of the percentages can be traced in Table IX.
It was observed that all patients (100\%) with depression, according to the PAD subscale, were correctly identified by the GDS-SF, indicating that the sensitivity of the scale is very high. On the other hand, $28.6 \%$ of patients (that based on the PAD depression subscale were included in the group of non-depressive) were wrongly identified by the GDS-SF as being at least susceptible to depression, fact that shows that the specificity of the scale is medium to high (more specifically, the specificity is $71.4 \%$ ). This confirms the authors' intention to use the instrument in clinical practice in case of the elderly who have a certain level of cognitive impairment as an auxiliary tool, it is rather an instrument that radiographs the situation and predicts the diagnosis than one that establishes it. There were no statistically significant differences concerning sex. Men presented similar levels of depression with those of women; trends towards difference appeared between patients diagnosed with neurocognitive disorder due to $\mathrm{AD}$, the score being slightly higher in men ( $M=9.66$ compared to $M=8.81$ ).

\section{Discussion}

There are few studies comparing the two types of neurocognitive disorders, and those that exist describe a rather poor methodology. It is clear why clinical groups are difficult to access for researchers; moreover, when talking about dementia, this factor makes it difficult for patients to participate in a possible study. Even if the groups that target the normal population cannot be compared quantitatively with the clinical groups, this should be compensated by a strict control of the parasitic variables by the researchers who choose to carry out their activity for this type of population. Without being proposed as a model of methodological rigor, the present study provides additional information regarding the differences that characterize the two types of neurocognitive disorders in terms of the psychological aspects affected.

Also, the results of the studies on this niche do not appear to be homogeneous. DeBettignies et al conclude from a comparison of the two types of neurocognitive disorders that AD patients had the lowest level of independent living, which significantly affected their quality of life and thus their well-being (18). Comparative studies on the size of affective distress are at the opposite end, suggesting that patients with vascular dementia have a higher level of behavioural disorder, anxiety, depression and language disorder $(19,20)$. Our data differ from the results of the studies mentioned, on each of the factors: quality of life, profile of emotional distress and perceived stress. Patients with neurocognitive disorder due to $\mathrm{AD}$ had a lower level of well-being, higher emotional distress and perceived stress compared to patients with neurocognitive disorder due to stroke. The reason why some experiments that 
comparatively study the two types of neurocognitive disorders presented results diametrically opposed to our study, may be due to the existence of distress due to cognitive causes and not to organic ones (this is the case of the neurocognitive disorder due to AD).

Even if the findings of this study do not seem to improve the unclear situation of the comparison in this area, there are some certain data obtained. For example, the existence of a high comorbidity between AD and depression is well known for a significant period of time in psychiatric and psychological studies. Summarizing several extensive studies, Aznar and Knudsen (21) showed that the prevalence of the elements of affective distress in the population with $\mathrm{AD}$ in the stage of severe deterioration is $15-17 \%$, whereas in the population with moderate cognitive deterioration the percentage increases vertically until $63 \%$. The decrease in the percentage of distress as cognitive impairment increases in severity can be explained by the awareness of the disease, which in turn decreases as the neurofibrillary tangles and plaques become larger.

The authors who studied the phenomenon of depression that occurs after stroke have called it post CVA (post-stroke) depression. One of the most important differences between depression in neurocognitive disorder due to $\mathrm{AD}$ and neurocognitive disorder due to stroke is precisely the factor that causes the neurocognitive disorder. If in the first case the organic element has a significant share (cortical changes), in the second case it is about the psychological causes that do not involve organic changes that directly cause depression. In other words, the causes of major depressive episode in patients with at least one stroke in the medical history are due to cognitions secondary to the stroke and the emotional states caused by it $(22,23)$. More explicitly, the phenomenology of major depressive episode that occurs after stroke is similar, almost identical to depression in people who have not suffered any traumatic brain injury (24).

It is observed in this way that people suffering from a neurocognitive disorder due to stroke can indeed manifest high levels of affective distress, but they do not have an organic cause, they may appear as any general medical condition that incapacitates or deprives the patient of certain things that contributed to his previous well-being and may at one time cause depressive symptoms. Hereby the understanding of the significant differences in level regarding perceived stress, negative dysfunctional emotions and perceived well-being between the two analysed groups, because the cause of depression in the $\mathrm{AD}$ group is an element influenced by specific cognitive deterioration through the definition of its phenomenology, fact that cannot be said in the case of the group of the patients with neurocognitive disorder due to stroke.

Regarding the difference in the perception of well-being in both types of patients, the negative correlation is edifying between neuropsychiatric symptoms (or the profile of affective distress) and well-being. Already arguing for the presence of organic depression in AD patients, it is somewhat natural to admit that they will have lower levels of subjectively perceived well-being compared to patients with neurocognitive disorder due to stroke. This is valid at least in the stage of disease onset and during the transition to moderate cognitive impairment, i.e. the categories covered by the present study.

A recent meta-analysis that followed 6 types of intervention focused on reducing the level of affective distress (in particular depression and anxiety) in people with dementia concluded that psychological treatments are effective in reducing the symptoms of distress (25-27). The post-intervention results showed that the level of depression in the group that benefited from the psychotherapeutic intervention $(\mathrm{M}=10.38)$ was much lower compared to the group that was not included in the program $(M=16.72)$. The data show the effectiveness of the intervention programs regarding the affective distress in the patients suffering from a form of the neurocognitive disorder. Due to this reason, it is indicated to maintain the proposal regarding the development of a specific structured intervention, especially on the types of neurocognitive disorders caused by $\mathrm{AD}$, respectively stroke.

The intention of the current research was to adapt the short form of GDS for the Romanian clinical population. Given that the intention of the authors who developed it was that the short form of the scale that initially had 30 items to be used in detecting depression in the elderly suffering from a cognitive disorder, specifying dementia, adapting this scale to the clinical Romanian population is considered to be a beneficial approach, especially for further research that aims to analyse the level of depression in the elderly, in the clinical context.

Even though the low number of study participants was motivated and highlighted the difference between research targeting the clinical population and those considering the normal population, the small group is a limitation. Also, there are certain variables that cannot be controlled, and which can interfere with the psychological factors analysed, such as the socio-family support that the patient benefits from. It is expected that a high level of socio-family support to correlate with a low level of emotional distress or perceived stress, or at least contribute to maintaining an optimal level of well-being. On the other hand, the low level of support from friends or family contributes to increasing the level of distress or may even cause it, which would interfere with the attempt to estimate the effect that the disease has on the patient's psychic.

The practical consequences of the results can be materialized by improving the existing therapeutic interventions for the two types of disorders, or they can contribute to the development of a new intervention with specific character, which will differentially treat the psychological consequences of the two pathologies. The intervention that can be developed through further research would increase the perceived level of well-being, respectively decrease the level of affective distress and stress perceived in both types of neurocognitive disorders. Another benefit is the knowledge of the subjective side of the disorders that have been studied, thus approaching psychiatric aspects from a psychological perspective.

In conclusion, the results of the present research reveal the higher level of dimension of affective distress in patients with neurocognitive disorder due to AD compared to patients diagnosed with neurocognitive disorder due to stroke. The same happens in the case of perceived stress, while the dimension of well-being is at the opposite pole, negatively correlating with the emotional distress. The data provided by this study thus support the theoretical studies that describe the cortical structures affected in AD and their implications on the psychological faculties of the patient. As a future research direction (which would focus on these types of neurocognitive disorders), the development of intervention programs is suggested, specifically focused on the affective characteristics of the two types 
of disorders. The results of this study in conjunction with other specialized studies may contribute to the improvement of the way in which the patient diagnosed with neurocognitive disorder due to AD or stroke is related to life and disease.

\section{Acknowledgements}

Not applicable.

\section{Funding}

No funding was received.

\section{Availability of data and materials}

All data generated or analyzed during this study are included in this published article.

\section{Authors' contributions}

CMD, DF, LP, AB, and MS selected the patients and analyzed and interpreted the data regarding the results of scales used for the patients' evaluation. All the authors substantially contributed to the conception of the research and the interpretation of the data, drafted the manuscript, and were major contributors in the writing of the manuscript. All authors read and approved the final version of the manuscript to be published.

\section{Ethics approval and consent to participate}

The study was conducted in accordance with the WMA Declaration of Ethical Helsinki - Medical Research Involving Human Principles for Subjects. All the participants provided a signed written informed consent and accepted to take part in the research. Ethics approval was received from the Medical Center Crucea Alba 'Dr Oros si asociatii', Oradea, Romania (no. 25/Sept. 11, 2019).

\section{Patient consent for publication}

Not applicable.

\section{Competing interests}

The authors declare that they have no competing interests.

\section{References}

1. Etters L, Goodall D and Harrison BE: Caregiver burden among dementia patient caregivers: A review of the literature. J Am Acad Nurse Pract 20: 423-428, 2008.

2. Wolstenholme J, Fenn P, Gray A, Keene J, Jacoby R and Hope T: Estimating the relationship between disease progression and cost of care in dementia. Br J Psychiatry 181: 36-42, 2002.

3. Uivarosan D, Bungau S, Tit DM, Moisa C, Fratila O, Rus M, Bratu OG, Diaconu CC and Pantis C: Financial burden of stroke reflected in a pilot center for the implementation of thrombolysis. Medicina (Kaunas) 56: 54, 2020.

4. Purza L, Abdel-Daim M, Belba A, Iovan C, Bumbu A, Lazar L, Bungau S and Tit DM: Monitoring the effects of various combination of specific drug therapies at different stages of Alzheimer's dementia. Farmacia 67: 477-481, 2019.
5. Mucke L: Alzheimer's disease. Nature 461: 895-897, 2009.

6. Kaerst L, Kuhlmann A, Wedekind D, Stoeck K, Lange P and Zerr I: Cerebrospinal fluid biomarkers in Alzheimer's disease, vascular dementia and ischemic stroke patients: A critical analysis. J Neurol 260: 2722-2727, 2013.

7. Fodor K, Tit DM, Pasca B, Bustea C, Uivarosan D, Endres L, Iovan C, Abdel-Daim MM and Bungau S: Long-term resveratrol supplementation as a secondary prophylaxis for stroke. Oxid Med Cell Longev 2018: 4147320, 2018.

8. Sabau M, Bungau S, Buhas CL, Carp G, Daina LG Judea-Pusta CT, Buhas BA, Jurca CM, Daina CM and Tit DM: Legal medicine implications in fibrinolytic therapy of acute ischemic stroke. BMC Med Ethics 20: 70, 2019.

9. Uivarosan D, Tit DM, Iovan C, Nistor-Cseppento DC, Endres L, Lazar L, Sava C, Sabau AM, Buhas C, Moleriu LC, et al: Effects of combining modern recovery techniques with neurotrophic medication and standard treatment in stroke patients. Sci Total Environ 679: 80-87, 2019.

10. Series H and Esiri M: Vascular dementia: A pragmatic review. Adv Psychiatr Treat 18: 372-380, 2012.

11. Jellinger KA: Pathology and pathogenesis of vascular cognitive impairment - a critical update. Front Aging Neurosci 5: 17, 2013.

12. Cohen D: The subjective experience of Alzheimer's disease: The anatomy of an illness as perceived by patients and families. Am J Alzheimer's Care Relat Disord Res 6: 6-11, 1991.

13. Stefan AM and Băban A: The Romanian version of the Geriatric Depression Scale: Reliability and validity. Cogn Brain Behav 21: 175-187, 2017.

14. Preoteasa CT and Preoteasa E: Psychometric properties of romanian version of who- 5 well-being index in dental students. Rom J Oral Rehabil 7: 21-27, 2015.

15. Opris D and Macovei M: The profile of emotional distress; Norms for the Romanian population. JEBP Psychother 7: 139-158, 2007.

16. Levenstein S, Prantera C, Varvo V, Scribano ML, Berto E, Luzi C and Andreoli A: Development of the Perceived Stress Questionnaire: A new tool for psychosomatic research. J Psychosom Res 37: 19-32, 1993.

17. Băban A (ed): Stress and Personality. Vol 1. 1st edition. Presa Universitară Clujeană, Cluj-Napoca, pp12-43, 1998 (In Romanian).

18. DeBettignies BH, Mahurin RK and Pirozzolo FJ: Insight for impairment in independent living skills in Alzheimer's disease and multi-infarct dementia. J Clin Exp Neuropsychol 12: 355-363, 1990

19. Fuh JL, Wang SJ and Cummings JL: Neuropsychiatric profiles in patients with Alzheimer's disease and vascular dementia. J Neurol Neurosurg Psychiatry 76: 1337-1341, 2005.

20. Sultzer DL, Levin HS, Mahler ME, High WM and Cummings JL: A comparison of psychiatric symptoms in vascular dementia and Alzheimer's disease. Am J Psychiatry 150: 1806-1812, 1993.

21. Aznar S and Knudsen GM: Depression and Alzheimer's disease: Is stress the initiating factor in a common neuropathological cascade? J Alzheimers Dis 23: 177-193, 2011.

22. Abdel-Daim MM, El-Tawil OS, Bungau SG and Atanasov AG: Applications of antioxidants in metabolic disorders and degenerative diseases: Mechanistic approach. Oxid Med Cell Longev 2019: 4179676, 2019.

23. Abdel-Daim MM, Zakhary NI, Aleya L, Bungău SG, Bohara RA and Siddiqi NJ: Aging, metabolic, and degenerative disorders: Biomedical value of antioxidants. Oxid Med Cell Longev 2018: 2098123, 2018

24. Narushima K and Robinson RG: Stroke-related depression. Curr Atheroscler Rep 4: 296-303, 2002.

25. Orgeta V, Qazi A, Spector A and Orrell M: Psychological treatments for depression and anxiety in dementia and mild cognitive impairment: Systematic review and meta-analysis. Br J Psychiatry 207: 293-298, 2015.

26. Roman-Filip C, Ungureanu A and Cernuşcă-Miţaru M: Painful tonic spasms and brainstem involvement in a patient with neuromyelitis optica spectrum disorder. Neurol Neurochir Pol 50: 55-58, 2016.

27. Ungureanu A, Rus L, Gligor F, Lazaroae A, Prodan L and Roman-Filip C: Intravenous levetiracetam as second line treatment for status epilepticus. Farmacia 64: 507-511, 2017. 